\title{
Type 2 diabetes mellitus as a risk factor for the onset of depression: a systematic review and meta-analysis
}

\author{
A. Nouwen • K. Winkley • J. Twisk • C. E. Lloyd • \\ M. Peyrot • K. Ismail • F. Pouwer • \\ for the European Depression in Diabetes (EDID) \\ Research Consortium
}

Received: 17 February 2010 / Accepted: 13 July 2010 /Published online: 14 August 2010

(C) The Author(s) 2010. This article is published with open access at Springerlink.com

\begin{abstract}
Aims/hypothesis An earlier meta-analysis showed that dia-
betes is a risk factor for the development and/or recurrence
of depression. Yet whether this risk is different for studies
using questionnaires than for those relying on diagnostic
Abstract
Aims/hypothesis An earlier meta-analysis showed that dia-
betes is a risk factor for the development and/or recurrence
of depression. Yet whether this risk is different for studies
using questionnaires than for those relying on diagnostic

Abstract
Aims/hypothesis An earlier meta-analysis showed that dia-
betes is a risk factor for the development and/or recurrence
of depression. Yet whether this risk is different for studies
using questionnaires than for those relying on diagnostic

Abstract
Aims/hypothesis An earlier meta-analysis showed that dia-
betes is a risk factor for the development and/or recurrence
of depression. Yet whether this risk is different for studies
using questionnaires than for those relying on diagnostic

Abstract
Aims/hypothesis An earlier meta-analysis showed that dia-
betes is a risk factor for the development and/or recurrence
of depression. Yet whether this risk is different for studies
using questionnaires than for those relying on diagnostic
\end{abstract}

Electronic supplementary material The online version of this article (doi:10.1007/s00125-010-1874-x) contains supplementary material, which is available to authorised users.

All authors are members of the European Depression in Diabetes (EDID) Research Consortium (www.edid-research.eu)

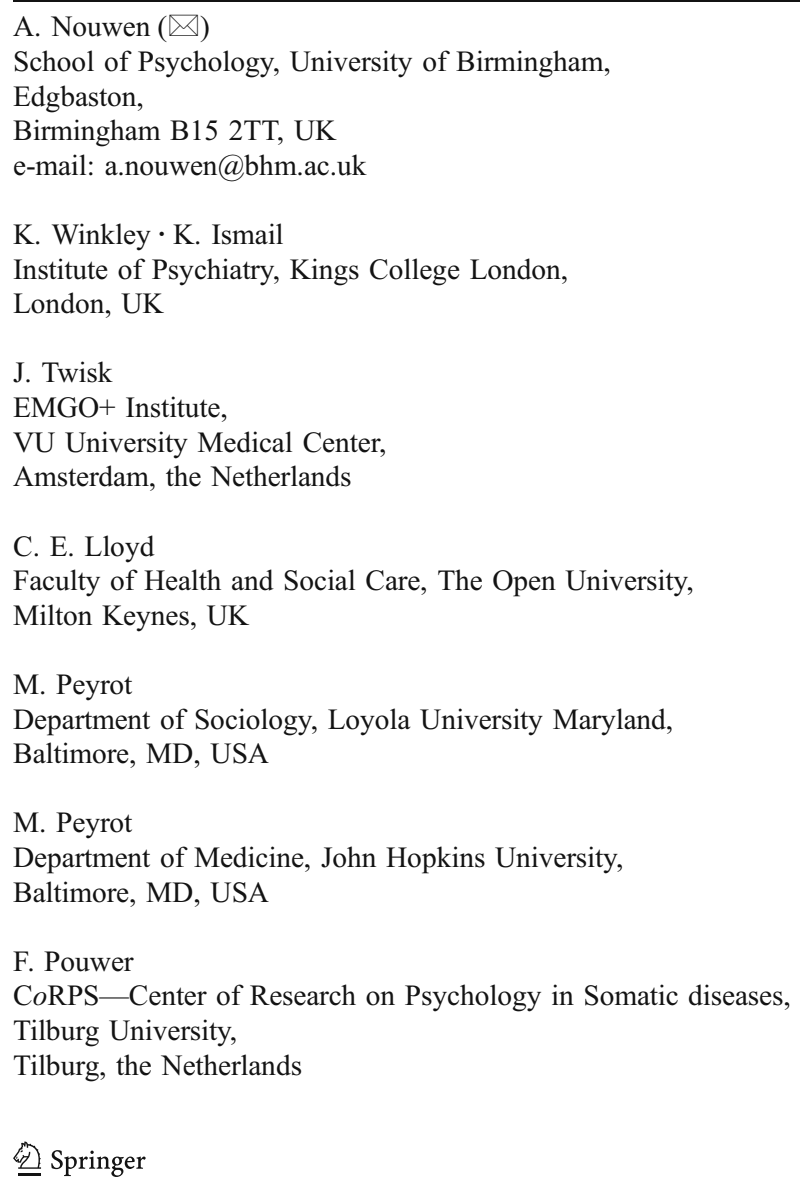

criteria for depression has not been examined. This study examined the association of diabetes and the onset of depression by reviewing the literature and conducting a meta-analysis of longitudinal studies on this topic.

Methods EMBASE, MEDLINE and PsycInfo were searched for articles published up to September 2009. All studies that examined the relationship between type 2 diabetes and the onset of depression were included. Pooled relative risks were calculated using fixed and random effects models.

Results Eleven studies met our inclusion criteria for this metaanalysis. Based on the pooled data, including 48,808 cases of type 2 diabetes without depression at baseline, the pooled relative risk was 1.24 (95\% CI 1.09-1.40) for the random effects model. This risk was significantly higher for studies relying on diagnostic criteria of depression than for studies using questionnaires. However, this difference was no longer significant when controlled for year of publication.

Conclusions/interpretation Compared with non-diabetic controls, people with type 2 diabetes have a $24 \%$ increased risk of developing depression. The mechanisms underlying this relationship are still unclear and warrant further research.

Keywords Critical review · Depression · Incidence . Meta-analysis $\cdot$ Systematic review Type 2 diabetes
Abbreviations
FEM Fixed effects model
MDD Major depressive disorder
REM Random effects model

\section{Introduction}

It is now commonly accepted that depressive symptoms and major depressive disorder (MDD) are twice as prevalent in individuals with type 2 diabetes $[1,2]$. Moreover, psycho- 
logical morbidity in people with diabetes poses huge challenges for clinical practice. In a recent WHO study, the greatest decrements in self-reported health were observed in those with both depression and diabetes, more so than in those with depression and other chronic conditions such as angina, arthritis or asthma [3].

Depression has been found to be associated with lower quality of life [4], poorer diabetes self-care [5], impaired glycaemic control [6], and an increased risk of developing diabetes-related complications [7]. Given the higher healthcare expenditure [8] and increased mortality $[9,10]$ associated with depression, it is therefore not surprising that clinical guidelines now recommend that all patients with diabetes undergo regular screening for depression $[11,12]$.

It remains unclear, however, whether the presence of diabetes increases risk for depression, or whether depression increases risk for diabetes. The early studies in this area used mainly cross-sectional study designs, which preclude any causal inferences as to the direction of this relationship, thus making any recommendations for practice problematic [1,2]. More recently, the temporal relationship between depression and diabetes has been the focus of a number of longitudinal prospective population-based studies. Three systematic reviews and meta-analyses [13-15] found that depression was associated with an increased risk of incident diabetes. The reverse, that diabetes may be a risk factor for depression, has been the focus of one earlier systematic review and meta-analysis, which included seven studies [15]. It was found that people with type 2 diabetes had a modest increased risk of developing depression. However, the authors did not distinguish between studies that used a diagnosis of a depressive disorder and those that measured depressive symptoms using self-report measures. This distinction is important, because high levels of depressive symptoms as measured through self-report questionnaires were found to be more reflective of general emotional and diabetes-specific distress than of clinical depression $[16,17]$. Therefore, this report examines the relationship between diabetes and depression by conducting a metaanalysis of longitudinal studies published on this subject in the peer-reviewed literature. Moreover, we examined separately studies that included a diagnosis of a depressive disorder and those using self-report measures of depression.

\section{Methods}

Retrieval of studies To identify the studies of interest, MEDLINE (1966 to September 2009), PsycInfo (1872 to September 2009), and EMBASE (1950 to September 2009) databases were searched. The search terms are shown in Electronic supplementary material (ESM) Table 1. Titles and abstracts of the retrieved studies were scanned to exclude studies that were clearly irrelevant. The full text of the remaining studies was then read by three authors (A. Nouwen, K. Winkley and F. Pouwer), who independently determined whether the studies met the inclusion criteria. Disagreements were solved by a fourth author (MP), who independently examined the studies, and then consensus was reached. Furthermore, the reference lists of articles that studied our topic of interest were scanned to check for additional publications.

Inclusion/exclusion criteria In this systematic review and meta-analysis, we included all studies that longitudinally examined the relationship between type 2 diabetes and onset of depression, irrespective of their study design. Studies were excluded if the authors did not explicitly exclude patients with prevalent depression at baseline or did not control for this statistically, and if there were insufficient data to estimate a relative risk (OR, risk ratio or HR). When multiple publications from the same study population were available, we included the most recent publication.

Data extraction Three authors (A. Nouwen, F. Pouwer and C. E. Lloyd) independently extracted data from the studies, in particular regarding: (1) name of first author, (2) publication year, (3) study design, (4) follow-up time in years, (5) number of patients in the analysis, (6) sex of patients, (7) age of patients, (8) method of depression assessment, (9) method of diabetes assessment, (10) unadjusted and adjusted relative risks and 95\% CI (adjusted for the largest number of confounders), (11) confounders adjusted for, (12) method used for exclusion of depressed patients at baseline, (13) overall incidence of depression per year.

In the included studies, method of depression assessment could be either (1) a diagnosis of depression assessed by a diagnostic psychiatric interview, (2) assessment of depressive symptoms by a self-reported questionnaire or (3) a diagnosis by a physician. Studies identified depressed patients at baseline through either self-report measures or screening, the latter using a psychiatric diagnostic interview for depression. Overall incidence per year was extracted as the crude incidence of depression in the whole study population, divided by follow-up duration. Type of diabetes could be assessed through either self-report or screening, i.e. measuring blood glucose of all patients, or from patients' medical records.

The quality of the included studies was rated independently by two authors (A. Nouwen, K. Winkley) using criteria for cohort studies from the Centre for Reviews and Dissemination, University of York, UK [18]. These include adequacy of the description of groups (type 2 diabetes mellitus, type 2 diabetes mellitus with depression, control without depression and control with depression), control for confounding variables (age, sex, socioeconomic status, education), blinding of baseline assessment data (diagnosis 
only), description of follow-up data, description of dropout rates, and comparison of dropout rates between groups on key variables. Any disagreement was independently reviewed by a third author (MP) until consensus was reached.

Statistical analysis Consistently with Mezuk et al. [15], in each study the relative risk of the model that most closely adjusted for demographic variables (age, sex, socioeconomic status, marital status) was used to estimate a pooled OR. Both the fixed effects model (FEM) and the random effects model (REM) were used. The fixed effects model assumes that variability between studies is exclusively due to random variation and individual studies are simply weighted by their precision. The random effects model assumes a different underlying effect for each study and takes this into consideration as an additional source of variation. A random effects meta-analysis is more conservative than a fixed effects meta-analysis, as it gives wider CIs around the point estimate, and is recommended when heterogeneity between studies exists [19]. Because of expected heterogeneity in the results, we report the results from only the REMs. A forest plot was made to show the relative risk and $95 \% \mathrm{CI}$ of each study and the pooled OR and $95 \%$ CI. Studies reporting HRs were also included. As the HR provides an underestimation of the OR [20] and the prevalence of depression is not very high, this underestimation would be small, resulting in a more conservative estimate of the pooled OR.

To provide visual assessment of publication bias a funnel plot was drawn, in which the OR was plotted on a logarithmic scale on the vertical axis against its corresponding standard error for each study on the horizontal axis. Asymmetry of the funnel plot is an indicator of publication bias. Publication bias was also assessed by means of the Begg-adjusted rank correlation test. To check the influence of publication bias, a pooled OR was calculated. A forest plot was used to visually assess homogeneity of the studies. Furthermore, this was tested with Cochran's Q test. Finally, the influence of several aspects of study design on incidence rates was investigated with meta-regression analysis. Because the number of studies in the meta-regression is small, we first conducted a set of bivariate regressions to identify significant correlates for inclusion in a multiple regression analysis. All statistical analyses were performed using STATA 10.0 (STATA Corporation, College Station, TX, USA).

\section{Results}

The MEDLINE search identified 385 articles, of which 10 [21-30] met our inclusion criteria and were subsequently included in the systematic review and meta-analysis (ESM
Fig. 1). The search in EMBASE (total number of studies= 381) identified one additional study [31] meeting the selection criteria, but no further studies were identified through the search in PsycInfo (total number of studies= 17). Thus, a total of 19 studies were retrieved for detailed evaluation, of which 11 studies were selected for inclusion in the meta-analysis. The extracted data of the 11 studies included are presented in ESM Table 2. It should be noted that two studies $[23,24]$ reported HRs rather than ORs.

Qualitative analysis The results of the quality assessment of the included studies can be found in ESM Table 3. Only one study [24] provided adequate description of the four groups (diabetes depressed, diabetes non-depressed, nondiabetes depressed, non-diabetes non-depressed) in terms of confounding variables such as age, sex, number and type of complications. As such, it was, in most cases, impossible to see whether the groups were comparable on important confounding variables. However, most studies $(n=8)$ controlled statistically for a number of potentially confounding variables.

Four studies used diagnostic criteria to determine depression status [24-26, 30], but none of these studies reported whether there was any blinding of the assessors to the status of diabetes. However, as Brown et al. [24] and O'Connor et al. [31] were retrospective studies and diagnosis of depression was made by general practitioners independent of the study rather than by trained assessors, lack of blinding was deemed less important. However, reliability of the diagnosis of depression in these two studies was not reported.

The follow-up time varied between less than 2 years [23] and 10 years [27]. Two studies [22, 26] combined data from waves at different time points ( 2 and 5 years follow-up) to calculate depression incidence. Finally, while all but two studies $[24,31]$ reported the proportion of the cohort that was followed up, only three studies [21, 22, 25] reported whether dropout rates and reasons for dropout were similar across groups. It should be noted that the criteria that were used for establishing diabetes differed among the studies, with most relying on doctor's diagnosis $[24,31]$ or selfreport of doctor's diagnosis [22, 23, 25-27]. Others also used medication use or blood tests such as OGTT or fasting plasma glucose [21, 27-30] or $\mathrm{HbA}_{1 \mathrm{c}}$ levels [27].

Meta-analysis Based on all studies, including 48,808 cases of type 2 diabetes, the pooled OR was 1.24 (95\% CI 1.09 1.40). The forest plot of the OR and $95 \%$ CI of each study, and the pooled OR of both the FEM and the REM, are shown in Fig. 1.

The funnel plot suggested possible publication bias (Fig. 2). This was also supported by Begg's adjusted rank correlation test $(p=0.70)$ and the Egger test $(p=0.81)$. 
Fig. 1 Forest plots showing the OR of depression in type 2 diabetes for individual studies using depression self-report questionnaires, diagnostic criteria, and all studies combined. Bars and diamonds indicate $95 \%$ CIs. The size of each square corresponds to the weight of the study in the meta-analysis. $\mathrm{D}+\mathrm{L}$, randomeffects estimate (Der Simonian and Laird method); I-V, fixedeffects estimate (inverse variance method)

\section{Study ID}

OR $(95 \% \mathrm{Cl})$

$\%$ Weight

$(\mathrm{I}-\mathrm{V})$

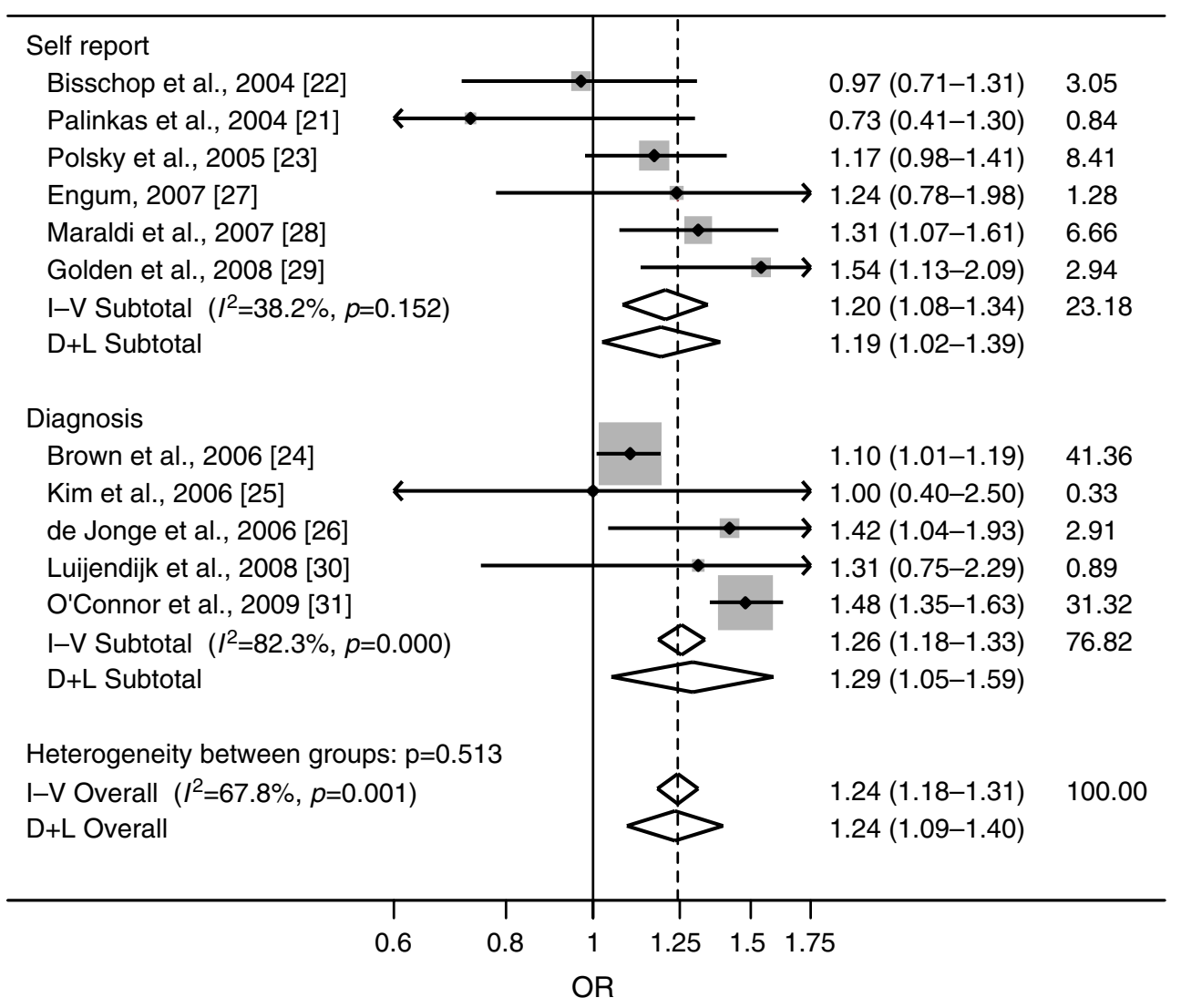

The test for heterogeneity was significant $(Q=30.84 ; d f=$ $10 ; p=0.001)$, with a moment-based estimate of between studies variance of 0.021 , indicating heterogeneity. We therefore stratified the studies by method of defining depression. Among the six studies that relied on questionnaires to define depression, the pooled OR was $1.19(95 \%$ CI 1.03-1.39) and the test for heterogeneity was not significant $(Q=8.03 ; d f=5 ; p=0.16)$, with a moment-based estimate of between studies variance of 0.013 . In the five studies that defined depression using diagnostic criteria, the

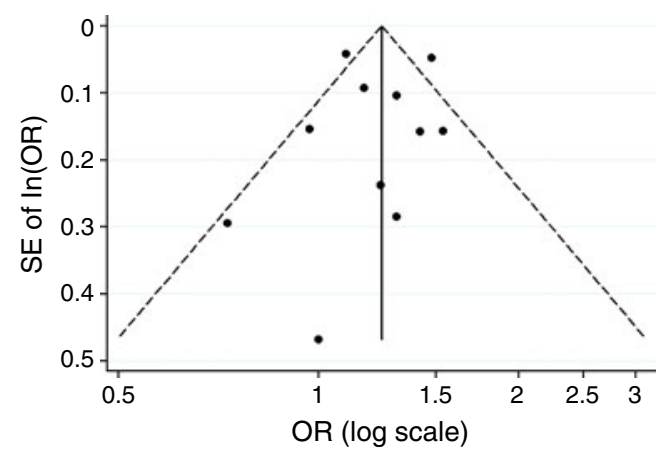

Fig. 2 Begg's funnel plot with pseudo $95 \%$ CI of all the studies included in the meta-analysis risk of depression was higher than for studies using questionnaires, with a pooled OR of 1.29 (95\% CI $1.05-$ 1.59). The test for heterogeneity was significant $(Q=22.42$; $d f=4 ; p<0.001$ ), with a moment-based estimate of between studies variance of 0.032. However, as one study [24] relying on diagnostic criteria to define depression included incident (new) cases of diabetes, the analysis was rerun without this study. The pooled OR for the four remaining studies was $1.47(95 \%$ CI 1.34-1.60) and the test for heterogeneity was not significant $(Q=0.91 ; d f=3 ; p=0.82)$, with a moment-based estimate of between studies variance of 0.00 .

Meta-regression Because stratifying by (controlling for) method of depression measurement (diagnosis or questionnaire) reduced the significant overall study heterogeneity to non-significance, meta-regression analysis was performed to examine this further. The results showed that type of depression measurement $(\beta=-0.25 ; p<0.001,95 \%$ CI $-0.37,-0.14)$ was a significant predictor of depression incidence, with rates higher for studies using diagnostic criteria to define depression.

Additional meta-analyses were performed to determine whether other factors might account for the heterogeneity in 
study-specific incidence rates. For example, as can be seen in Fig. 1, the forest plot showed that the ORs increase over time, suggesting that the incidence of depression is increased in more recent studies. Therefore, a set of single-factor meta-regression analyses was performed with time since publication, follow-up time, number of followup depression measurements ( 1 vs $>1$ or continuous assessment), sample size, and number of people with diabetes as separate predictors of depression incidence OR. The results showed that year of publication $(\beta=0.09$; $p<0.001,95 \%$ CI $0.05-0.12$ ) was a significant predictor of depression incidence OR, but time of follow-up, sample size, the number of people with diabetes, and number of follow-up depression measurements were not $(p>0.09)$. Because type of depression measurement and year of publication may be confounded, we also examined these together in a single regression model. The results showed that year of publication remained significant $(\beta=0.09$, $p<0.02,95 \%$ CI $0.02-0.16)$ but type of depression measurement was no longer significant $(\beta=-0.07, p<0.95$, $95 \%$ CI $-0.24-0.22$ ). The results did not change when repeated without Brown et al. [24].

\section{Discussion}

The results of this systematic review and meta-analysis of 11 studies involving 172,521 participants, including 48,808 people with type 2 diabetes, show that overall people with type 2 diabetes have a $24 \%$ increased risk of incident depression compared with people without diabetes. The risk found in the current study including four more studies is slightly higher than the one found in an earlier metaanalysis (OR 1.15, 95\% CI 1.02-1.30) [15]. When studies that used questionnaires to define depression were separated from those using diagnostic criteria, the odds of incident depressive symptoms were somewhat lower (OR 1.21) than the risk of developing incident depressive disorder (OR 1.29). Interestingly, this difference was accentuated when a study that included incident diabetes rather than prevalent diabetes [24] was excluded from the analysis; the increased chance of people with diabetes developing a depressive disorder was almost $50 \%$. Although the difference in incidence rates between diagnostic studies and questionnaire studies was significant, the meta-regression analysis showed that this effect may have been confounded by higher ORs in more recent published studies.

Relative to the earlier meta-analysis [15], heterogeneity was larger in the present meta-analysis. This is primarily a function of the fact that later studies, those not included in the earlier analysis, reported higher levels of increased risk for persons with diabetes. We have no definitive explana- tion for the apparent increase in incident depression in more recent studies, although it is possible that both people with diabetes and healthcare professionals have become increasingly aware of symptoms of depression in diabetes, which may have led to higher scores at follow-up measurements.

The results of this study should be interpreted cautiously with regard to whether diabetes is a risk for incident depression in the strictest sense, i.e. the initial episode of depression. With the exception of Brown et al. [24], who excluded participants with depressive episodes within 3 years before the onset of the study, none of the other studies in this meta-analysis excluded people who had had depression at some point during a lengthy period before the onset of the study. Because recurrence of depressive disorder is high, especially among people with diabetes [32], the increased incidence of depressive episodes may be due, in part, to diabetes or stressors related to diabetes triggering depression more frequently in people with vulnerability for depression, i.e., people with a history of depression.

It is possible that the incident depression was associated with the development of diabetes-related complications. For example, the Brown et al. [24] study only included people with incident diabetes, and the likelihood of diabetesrelated complications is likely to be lower in this sample. Interestingly, in this study the risk of developing depression was increased in people with cerebrovascular disease and peripheral arterial disease but not in those with coronary artery disease. de Jonge et al. [26] found that the longitudinal association of diabetes and depression was reduced after controlling for other chronic somatic diseases and was no longer significant. These findings are consistent with the results of two large cross-sectional studies [33, 34] showing that the prevalence of depressive disorder was higher in diabetes patients with diabetes-related complications, especially cardiovascular disease, than in those without complications.

The increased incidence rate for depression in people with type 2 diabetes found in the current meta-analysis (OR 1.19- 1.47) contrasts with a two-fold increase in the prevalence rate of depression (OR 2.0) found in a previous meta-analysis [1]. This is likely to be a result of two distinct processes. First, because prevalence is a function of not only incidence but also duration, the prevalence of a chronic condition such as depression is higher than one might expect based on the incidence rate alone. This line of reasoning is supported by earlier reports that, among people with diabetes, episodes of MDD last longer and are more recurrent $[32,35]$. Our finding that the risk in diabetes of developing depressive disorder is greater than that of developing depressive symptoms is consistent with this. In addition, because depression is a risk factor for the development of diabetes [13], relatively more people with 
diabetes would have a history of depression and as such an increased risk of developing a further episode.

This study has several limitations. First, measures of depression varied among studies. We were able to examine several aspects of this variation, finding that few factors were associated with study-specific levels of diabetes risk. However, there was substantial variation among studies using a particular form of depression measurement. Among the studies that used diagnostic criteria for the assessment of depression, two relied on the clinical judgement of general practitioners to establish a diagnosis while the other two used formal diagnostic interview schedules with trained researchers. Among studies using self-report questionnaires to assess depressive symptoms, different questionnaires and different cut-off values were used to define cases of depression. Because self-reported depressive symptoms in people with diabetes have been found to be indicative of general emotional and diabetes-specific distress rather than depressive disorder [16], the questionnaire studies, especially those with lower cut-off values, may have resulted in an over-estimation of the incidence rate of clinically significant levels of depression in people with diabetes. In addition, some studies also included antidepressant medication use as a stand-alone proxy measure of depression [24, 28, 29, 31]. However, because antidepressant medication is prescribed for other problems, such as neuropathic pain [36, 37] or sedation, this also may have artificially increased the incidence rate, particularly among people with diabetes. Finally, a number of studies [21, 27] used different measures or cut-off values at baseline than at follow-up, which may have led to misclassification of cases of depression at baseline and therefore affected the incidence rates for depression.

Another limitation is that the majority of the studies analysed here limited the assessment of incident depression to a single follow-up at a distinct time point, and none assessed the onset and offset of depressive episodes; thus these studies provide no insight into the temporal dynamics of the episodes.

Finally, the relatively small number of studies available for analysis limited our ability to examine the independent contribution of multiple methodological factors in accounting for the between-study variation in diabetes-related risk for depression.

\section{Conclusion}

Overall, the results from this meta-analysis suggest that the risk of a new depressive episode is higher among people with type 2 diabetes than in the general population. This increased risk may be greater among people who have suffered a previous depressive episode or who have diabetes-related complications. Moreover, the risk of incident depression associated with diabetes appears to be increasing over time. Because of the negative consequences of depression in diabetes, such as poorly controlled diabetes [6], an increased risk of complications [7], higher mortality [10] and increased healthcare costs [8], it is important that these vulnerable groups should be screened regularly for depression. We would further suggest that such efforts should be incorporated into treatment programmes for depression [38].

Acknowledgements The authors would like to thank A. Kokoszka (Department of Psychiatry, Medical University Warsaw, Poland), N. Hermanns (Forschungsinstitut Diabetes-Akademie \& Diabetes Klinik Bad Mergentheim, Germany) and J. Assies (Department of Psychiatry, Academic Medical Centre, University of Amsterdam, the Netherlands) for their comments and suggestions on an earlier version of the manuscript.

Duality of interest The authors declare that there is no duality of interest associated with this manuscript.

Open Access This article is distributed under the terms of the Creative Commons Attribution Noncommercial License which permits any noncommercial use, distribution, and reproduction in any medium, provided the original author(s) and source are credited.

\section{References}

1. Anderson RJ, Freedland KE, Clouse RE, Lustman PJ (2001) The prevalence of comorbid depression in adults with diabetes: a meta-analysis. Diabetes Care 24:1069-1078

2. Ali S, Stone MA, Peters JL, Davies MJ, Khunti K (2006) The prevalence of co-morbid depression in adults with Type 2 diabetes: a systematic review and meta-analysis. Diabet Med 23:1165-1173

3. Moussavi S, Chatterji S, Verdes E, Tandon A, Patel V, Ustun B (2007) Depression, chronic diseases, and decrements in health: results from the World Health Surveys. Lancet 379:851-858

4. Schram MT, Baan CA, Pouwer F (2009) Depression and quality of life in patients with diabetes: a systematic review from the European depression in diabetes (EDID) research consortium. Curr Diabetes Rev 5:112-119

5. Gonzalez JS, Peyrot M, McCarl LA et al (2008) Depression and diabetes treatment nonadherence: a meta-analysis. Diabetes Care 31:2398-2403

6. Lustman PJ, Anderson RJ, Freedland KE, de Groot M, Carney RM, Clouse RE (2000) Depression and poor glycemic control: a meta-analytic review of the literature. Diabetes Care 23:934-942

7. de Groot M, Anderson R, Freedland KE, Clouse RE, Lustman PJ (2002) Association of depression and diabetes complications: a meta-analysis. Psychosom Med 63:619-630

8. Egede LE, Zheng D, Simpson K (2002) Comorbid depression is associated with increased health care use and expenditures in individuals with diabetes. Diabetes Care 25:464-470

9. Bruce DG, Davis WA, Starkstein SE, Davis TM (2005) A prospective study of depression and mortality in patients with type 2 diabetes: the Fremantle Diabetes Study. Diabetologia 48:2532-2539 
10. Katon WJ, Rutter C, Simin G et al (2005) The association of comorbid depression with mortality in patients with type 2 diabetes. Diabetes Care 28:2668-2672

11. International Diabetes Federation (2005) Global guideline for type 2 diabetes. International Diabetes Federation. Available from www.idf.org/Global_guideline, accessed 28 January 2010

12. National Institute for Health and Clinical Excellence. Depression: management of depression in primary care and secondary care. National Institute for Health and Clinical Excellence. Available from http://guidance.nice.org.uk/CG23, accessed 28 January 2010

13. Knol MJ, Twisk JW, Beekman AT, Heine RJ, Snoek FJ, Pouwer F (2006) Depression as a risk factor for the onset of type 2 diabetes mellitus. A meta-analysis. Diabetologia 49:837-845

14. Cosgrove MP, Sargeant LA, Griffin SJ (2008) Does depression increase the risk of developing type 2 diabetes? Occup Med (Lond) 58:7-14

15. Mezuk B, Eaton WW, Albrecht S, Golden SH (2008) Depression and type 2 diabetes over the lifespan: a meta-analysis. Diabetes Care 31:2383-2390

16. Fisher L, Skaff MM, Mullan JT et al (2007) Clinical depression vs distress among patients with type 2 diabetes: not just a question of semantics. Diabetes Care 30:542-548

17. Fisher L, Mullan JT, Arean P, Glasgow RE, Hessler D, Masharani U (2010) Diabetes distress but not clinical depression or depressive symptoms is associated with glycemic control in both cross-sectional and longitudinal analyses. Diabetes Care 33:23-28

18. Criteria for cohort studies from the Centre for Reviews and Dissemination, University of York. Available from www.york.ac.uk/ inst/crd/CRD_Reports/crdreport4_ph5.pdf, accessed 29 January 2010

19. Fleiss JL, Gross AJ (1991) Meta-analysis in epidemiology, with special reference to studies of the association between exposure to environmental tobacco smoke and lung cancer: a critique. J Clin Epidemiol 44:127-139

20. Symons MJ, Moore DT (2002) Hazard ratio and prospective epidemiological studies. J Clin Epidemiol 55:893-899

21. Palinkas LA, Lee PP, Barrett-Connor E (2004) A prospective study of type 2 diabetes and depressive symptoms in the elderly: the Rancho Bernardo Study. Diabet Med 21:1185-1191

22. Bisschop MI, Kriegsman DM, Deeg DJ, Beekman AT, van Tilburg W (2004) The longitudinal relation between chronic diseases and depression in older persons in the community: the Longitudinal Aging Study Amsterdam. J Clin Epidemiol 57: 187-194

23. Polsky D, Doshi JA, Marcus S et al (2005) Long-term risk for depressive symptoms after a medical diagnosis. Arch Intern Med 165:1260-1266
24. Brown LC, Majumdar SR, Newman SC, Johnson JA (2006) Type 2 diabetes does not increase risk of depression. CMAJ 175:42-46

25. Kim JM, Stewart R, Kim SW, Yang SJ, Shin IS, Yoon JS (2006) Vascular risk factors and incident late-life depression in a Korean population. Br J Psychiatry 189:26-30

26. de Jonge P, Roy JF, Saz P, Marcos G, Lobo A, ZARADEMP Investigators (2006) Prevalent and incident depression in community-dwelling elderly persons with diabetes mellitus: results from the ZARADEMP project. Diabetologia 49:2627-2633

27. Engum A (2007) The role of depression and anxiety in onset of diabetes in a large population-based study. J Psychosom Res 62:31-38

28. Maraldi C, Volpato S, Penninx BW et al (2007) Diabetes mellitus, glycemic control, and incident depressive symptoms among 70 - to 79-year-old persons: the health, aging, and body composition study. Arch Intern Med 167:1137-1144

29. Golden SH, Lazo M, Carnethon M et al (2008) Examining a bidirectional association between depressive symptoms and diabetes. JAMA 299:2751-2759

30. Luijendijk HJ, Stricker BH, Hofman A, Witteman JC, Tiemeier H (2008) Cerebrovascular risk factors and incident depression in community-dwelling elderly. Acta Psychiatr Scand 118:139-148

31. O'Connor PJ, Crain AL, Rush WA, Hanson AM, Fischer LR, Kluznik JC (2009) Does diabetes double the risk of depression? Ann Fam Med 7:328-335

32. Lustman PJ, Griffith LS, Clouse RE (1988) Depression in adults with diabetes. Results of 5-yr follow-up study. Diabetes Care 11:605-612

33. Pouwer F, Beekman AT, Nijpels G et al (2003) Rates and risks for co-morbid depression in patients with Type 2 diabetes mellitus: results from a community-based study. Diabetologia 46:892-898

34. Peyrot M, Rubin RR (1997) Levels and risks of depression and anxiety symptomatology among diabetic adults. Diabetes Care 20:585-590

35. Lustman PJ, Griffith LS, Freedland KE, Clouse RE (1997) The course of major depression in diabetes. Gen Hosp Psychiatry 19:138-143

36. Edwards JL, Vincent AM, Cheng HT, Feldman EL (2008) Diabetic neuropathy: mechanisms to management. Pharmacol Ther 120:1-34

37. Hall GC, Carroll D, McQuay HJ (2008) Primary care incidence and treatment of four neuropathic pain conditions: a descriptive study, 2002-2005. BMC Fam Pract 9:26

38. Pouwer F (2009) Should we screen for emotional distress in type 2 diabetes mellitus? Nat Rev Endocrinol 5:665-671 